\title{
Effects of interplanetary transport on derived energetic particle source strengths
}

\author{
E. E. Chollet, ${ }^{1,2}$ J. Giacalone, ${ }^{2}$ and R. A. Mewaldt ${ }^{1}$ \\ Received 4 September 2009; revised 7 December 2009; accepted 13 January 2010; published 2 June 2010.
}

[1] We study the transport of solar energetic particles (SEPs) in the inner heliosphere in order to relate observations made by an observer at $1 \mathrm{AU}$ to the number and total energy content of accelerated particles at the source, assumed to be near the Sun. We use a numerical simulation that integrates the trajectories of a large number of individual particles moving in the interplanetary magnetic field. We model pitch angle scattering and adiabatic cooling of energetic ions with energies from $50 \mathrm{keV}$ nucleon ${ }^{-1}$ to $100 \mathrm{MeV}$ nucleon $^{-1}$. Among other things, we determine the number of times that particles of a given energy cross $1 \mathrm{AU}$ and the average energy loss that they suffer because of adiabatic deceleration in the solar wind. We use a number of different forms of the interplanetary spatial diffusion coefficient and a wide range of scattering mean-free paths and consider a number of different ion species in order to generate a wide range of simulation results that can be applied to individual SEP events. We apply our simulation results to observations made at $1 \mathrm{AU}$ of the 20 February 2002 solar energetic particle event, finding the original energy content of several species. We find that estimates of the source energy based on SEP measurements at 1 AU are relatively insensitive to the mean-free path and scattering scheme if adiabatic cooling and multiple crossings are taken into account.

Citation: Chollet, E. E., J. Giacalone, and R. A. Mewaldt (2010), Effects of interplanetary transport on derived energetic particle source strengths, J. Geophys. Res., 115, A06101, doi:10.1029/2009JA014877.

\section{Introduction}

[2] The acceleration of energetic charged particles and cosmic rays is one of the most important problems in solar physics, space physics, and astrophysics. The Sun and heliosphere provide a laboratory where acceleration processes may be studied in detail. In situ measurements carried out on spacecraft in the heliosphere and remote high-resolution observations of the Sun are providing many new insights into these problems. It is generally believed that the largest fluxes of high-energy charged particles seen in our solar system result from violent solar activity due to the reconfiguration of large-scale magnetic fields on the Sun. This process can release an enormous amount of energy in various forms, such as X-rays, $\gamma$-rays, and heating of the surrounding plasma. These solar explosions can also lead to coronal mass ejections (CMEs) and create large-scale shock waves which accelerate charged particles to high energies. The accelerated, fast moving particles usually arrive at Earth minutes to hours after the event.

[3] Though the total energy of a large solar event can be estimated to an order of magnitude, how much of this energy goes into energetic particles that escape into inter-

\footnotetext{
${ }^{1}$ Space Radiation Laboratory, California Institute of Technology, Pasadena, California, USA.

${ }^{2}$ Lunar and Planetary Laboratory, University of Arizona, Tucson, Arizona, USA.

Copyright 2010 by the American Geophysical Union. 0148-0227/10/2009JA014877
}

planetary space remains an open question. Emslie et al. [2004] described studies aimed at estimating the energy budget in two large events in 2002. The authors pointed out that the amount of energy needed to accelerate interplanetary energetic particles is uncertain by a factor of several, partly because particles likely scatter across 1 AU multiple times. Li et al. [2003] studied the effect on the measured fluence for particles at a CME shock propagating outward from $0.1 \mathrm{AU}$, noting the number of times a particle crossed $1 \mathrm{AU}$ as a function of energy. Mewaldt et al. [2005a, 2005b] compared the energy content of CMEs and accelerated particles in a number of large solar energetic particle (SEP) events, including corrections for multiple crossings, and found that the energy in energetic particles is a significant fraction of the CME kinetic energy.

[4] In this work, we perform a number of numerical simulations over a wide variety of parameters (scattering mean-free path, particle energy, ion species, etc.) in order to compile a range of results that can be used to relate observations made at $1 \mathrm{AU}$ to the number of accelerated particles and their total energy at the source. We then apply our results to one particular example of a SEP event. In our model, we assume that the particles are released impulsively near the Sun, which is representative of the physics of particles accelerated over a short period of time. These particles then travel through space along the (assumed Parker spiral) interplanetary magnetic field, where they are observed by spacecraft at $1 \mathrm{AU}$. We illustrate two major considerations that should be taken into account in evalu- 
ating the efficiency of particle acceleration: multiple crossings of an observing location and energy loss during transit from the source.

[5] A description of our simulation model is presented in section 2, and the results are presented in section 3. Section 4 applies these results to an example of a SEP event observed during solar cycle 23 and derives the original source strengths for various species.

\section{Numerical Model}

[6] In order to determine the number of times particles cross Earth's orbit, we must solve for the motions of individual charged particles. The same information cannot be obtained by solving the energetic particle transport equation [Parker, 1965], which provides the distribution function itself rather than information about any given particle. Thus, we need to use an approach that computes the orbits of individual particles. Our model does this by integrating the equations of motion for a large number of ions moving under the influence of forces arising from the large-scale heliospheric electromagnetic field. This model allows for large anisotropies to exist, and all basic transport effects are included.

[7] The heliospheric magnetic field is assumed to be the nominal Parker spiral, given by

$$
\mathbf{B}=B_{0}\left(\frac{\mathrm{r}_{0}}{\mathrm{r}}\right)^{2} \hat{\mathrm{r}}-B_{0}\left(\frac{\mathrm{r}_{0}}{r}\right) \frac{\mathrm{r}_{0} \Omega_{\odot} \sin \theta}{V_{w}} \hat{\phi},
$$

where $B_{0}$ is the strength of the open magnetic field at the base, $\mathrm{r}=\mathrm{r}_{0}$ (assumed here to be the radius of the Sun), $\Omega_{\odot}$ is the solar rotational frequency, $V_{w}$ is the solar wind speed, and $\mathrm{r}$ and $\theta$ define the usual spherical coordinate system. For all simulations presented in this paper, we use the following values: $B_{0}=1.6 \mathrm{G}, \Omega_{\odot}=2.8 \times 10^{-6} \mathrm{~s}^{-1}$, and $V_{w}=4 \times$ $10^{7} \mathrm{~cm} \mathrm{~s}^{-1}$.

[8] Our Fortran code begins with an impulsive, isotropic, monoenergetic release of $10^{4}$ nuclei at each of 30 energies at $0.05 \mathrm{AU}$ (approximately $11 R_{\odot}$ ). We included initial particle energies between $50 \mathrm{keV}$ nucleoo ${ }^{-1}$ and $100 \mathrm{MeV}$ nucleon $^{-1}$ for five particle species: protons, helium and carbon together, oxygen, and iron, which are assumed to have mass-to-charge ratios $(M / Q)$ of 1.0, 2.0, 2.67, and 4.0, respectively. Because the code is nonrelativistic, we did not consider higher energies. These energies and species are particularly useful since a great deal of data are available in the energy range from a few tens of $\mathrm{keV}$ nucleon $^{-1}$ to $\sim 100 \mathrm{MeV}$ nucleon $^{-1}$ from experiments such as the Ultra Low Energy Isotope Spectrometer (ULEIS), Solar Isotope Spectrometer (SIS), and Electron, Proton, and Alpha Monitor (EPAM) instruments on the ACE spacecraft, the Solar and Heliospheric Observatory Energetic and Relativistic Nuclei and Electron (SOHO/ERNE) and Solar Anomalous and Magnetospheric Particle Explorer (SAMPEX) ProtonElectron Telescope (PET) instruments, the Suprathremal Energetic Particle (STEP) and Low Energy Matrix Telescope (LEMT) instruments on Wind, and the Energetic Particle Sensor (EPS) instrument on NOAA's GOES satellites.

[9] The trajectories are computed by solving the equations of motion for each charged particle. In order to save computational time, we treat the particles adiabatically. That is, for relatively short time periods (much shorter than the mean time between particle scatterings), but still much larger than the cyclotron period, we assume the perpendicular kinetic energy over the magnetic field magnitude $\left(W_{\perp} / B\right)$ is constant in the frame corotating with the Sun. Also, in this frame, the particle kinetic energy is conserved. This allows us to determine the resulting kinetic energy and pitch angle in the local solar wind frame through the appropriate frame transformations. This scheme has been compared to runs where the equations of motion are integrated directly, including the gyromotion, and has been found to be in excellent agreement. The adiabatic scheme is simply much faster. Note also that our model includes adiabatic deceleration in the expanding solar wind, pitch angle focusing, and pitch angle scattering, including cross-field diffusion (in the form of hard sphere scattering), but this is not important for the purposes of this paper.

[10] The effect of small-scale magnetic irregularities, which act to scatter the particles, is included by using an ad hoc scattering algorithm. At each time step of the numerical integration of a given particle trajectory, a particle is tested for scattering by comparing a random number (between 0 and 1) with a probability of scattering. This is given by $\mathcal{P}_{s}=\Delta t / \tau(\mathrm{r}, E)$, where $\Delta t$ is the time step and $\tau(\mathrm{r}, E)$ is the mean scattering time at the position $\mathrm{r}$ and particle energy $E$. We consider three different forms for $\tau$, which we relate to the scattering mean-free path parallel to the magnetic field by $\lambda_{\|}=v \tau$, where $v$ is the particle speed in the local plasma frame.

[11] In the simplest scheme, the particles scatter with a mean-free path that is spatially constant and proportional to the particle mass-to-charge ratio, i.e.,

$$
\lambda_{0}=\lambda_{i} \frac{M}{Q} .
$$

The variable $\lambda_{i}$ for protons varies from 0.01 to $1 \mathrm{AU}$, which covers the range considered typical for particles in the inner heliosphere [Bieber et al., 1994], as well as 10 AU (essentially scatter-free propagation).

[12] The second scattering scheme is similar to that outlined by Li et al. [2003, section 5.3] and is given by

$$
\lambda=\lambda_{0}\left(\frac{p c}{1 \mathrm{GeV}}\right)^{\alpha}\left(\frac{\mathrm{r}}{1 \mathrm{AU}}\right)^{\beta},
$$

where $\lambda_{0}$ is an input parameter that is varied ( $0.4 \mathrm{AU}$, $0.8 \mathrm{AU}$, and 1.6 AU). In our calculations, we use $\lambda_{0}$ from equation (1), $\alpha$ is taken to be 0 , and $\beta$ is taken to be $2 / 3$, though $\mathrm{Li}$ et al. [2003] consider other cases. The goal is not to make a direct comparison with $\mathrm{Li}$ et al. [2003] but rather to illustrate the difference between the case in which $\lambda_{\|}$is constant and one in which $\lambda_{\|}$increases with distance from the Sun.

[13] The final scheme assumes a form given by the quasilinear theory [Jokipii, 1966] given by

$$
\lambda_{\mathrm{QLT}}(\mathrm{r}, v)=\lambda_{i}\left(\frac{v(\mathrm{r})}{v_{0}}\right)^{1 / 3}\left(\frac{B(1 \mathrm{AU})}{B(\mathrm{r})}\right)^{1 / 3}\left(\frac{M}{Q}\right)^{-1 / 3},
$$

where $\lambda_{i}$ is the mean-free path at $1 \mathrm{AU}$ for a proton moving with speed $v_{0}$, taken to be that of a proton with an energy of $1 \mathrm{MeV}$. 


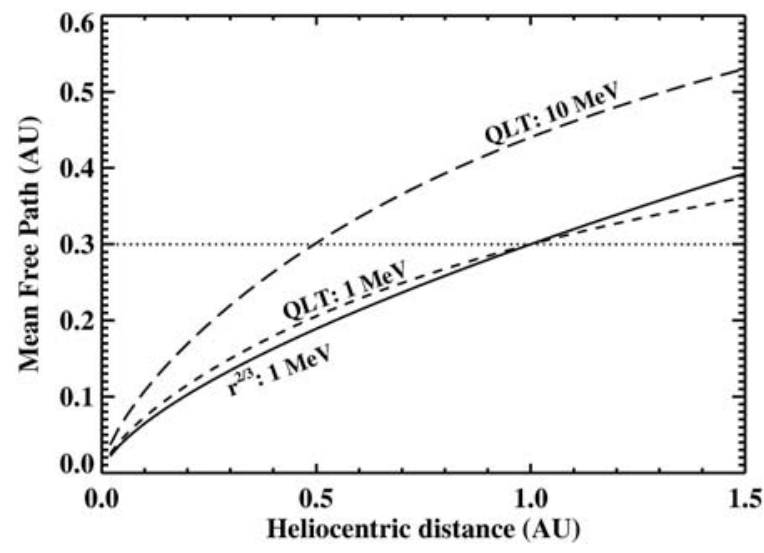

Figure 1. Simulation mean-free path for protons as a function of distance, normalized to $0.3 \mathrm{AU}$ at $1 \mathrm{AU}$. The dashed lines are the quasilinear scheme (long is $10 \mathrm{MeV}$ and short is $1 \mathrm{MeV}$ ), the solid line is the distance-dependent scheme, and the dotted line is the constant mean-free path.

[14] A plot of the mean-free path as a function of $r$ is presented in Figure 1 for protons using each of these schemes, assuming $\lambda_{i}$ is $0.3 \mathrm{AU}$. Two instances of the quasilinear scheme are plotted, one for a $1 \mathrm{MeV}$ proton and one for a $10 \mathrm{MeV}$ proton. The quasilinear $1 \mathrm{MeV}$ case and $r^{2 / 3}$ case differ only slightly, since the radial dependence in the quasilinear case comes from the Parker spiral magnetic field, and an $\mathrm{r}^{2 / 3}$ dependence arises if we only consider the radial part of the Parker spiral field. Each particle is followed for 10 days or until it crosses $10 \mathrm{AU}$, whichever comes first. Each time a particle crosses $1 \mathrm{AU}$, we note the time, its kinetic energy per nucleon, and the original particle energy.

\section{Results}

[15] Figure 2a shows the number of crossings as a function of energy, mean-free path, and species, and Figure $2 b$ shows the fraction of the original energy that remains when the particle crosses $1 \mathrm{AU}$ as a function of mean-free path, original energy, and species. The results in Figure 2 are for the constant mean-free path scattering scheme. The mean-free path largely determines the resulting energy loss and number of times a given particle crosses $1 \mathrm{AU}$. For the shortest mean-free path of 0.01 AU the particles can lose $70 \%-90 \%$ of their energy and can cross more than 10 times. For the longest mean-free path of $10 \mathrm{AU}$, the particles lose almost none of their original energy and only cross once. The small energy loss at this mean-free path is likely an artifact of the isotropic release of particles. When the particles are beamed along the field, this small energy loss goes away.

[16] For the constant mean-free path scheme, the original energy of the particles dominates the number of crossings, and the mass-to-charge ratio of the species chosen makes a difference by a factor of several in both number of crossings and energy loss. From the lowest to highest original energies, the number of crossings can increase by more than a factor of 10 for the shortest mean-free path while only increasing by a factor of 2-3 for a $1 \mathrm{AU}$ mean-free path and not substantially increasing at all for scatter-free propagation. For a given scattering mean-free path and species, particles with higher original energy cross a larger number of times and lose a smaller fraction of their energy than particles with lower original energy. Particles with larger mass-to-charge ratios cross fewer times and lose a smaller fraction of their original energy.

[17] Figure 3 is a graph of the fraction of the originally injected particles that cross a given number of times for three constant mean-free paths. A mean-free path of 10 AU (black) is essentially scatter-free, while the meanfree paths of $0.1 \mathrm{AU}$ (red) and $1 \mathrm{AU}$ (blue) bracket the range of commonly used values. The particles in this plot are protons with an original energy of $1.5 \mathrm{MeV}^{\text {nucleon }}{ }^{-1}$, around the middle of the range we studied, and the results presented here are representative of all species. When the propagation is close to scatter-free, nearly $100 \%$ of the particles cross exactly once. At progressively shorter meanfree paths, the distribution gets flatter. Roughly $0.1 \%$ of the particles cross 10 or more times if the mean-free path is $1 \mathrm{AU}$, and roughly $8 \%$ of the particles cross 10 or more times if the mean-free path is 0.1 AU. Most of the particles cross an odd number of times because almost all particles
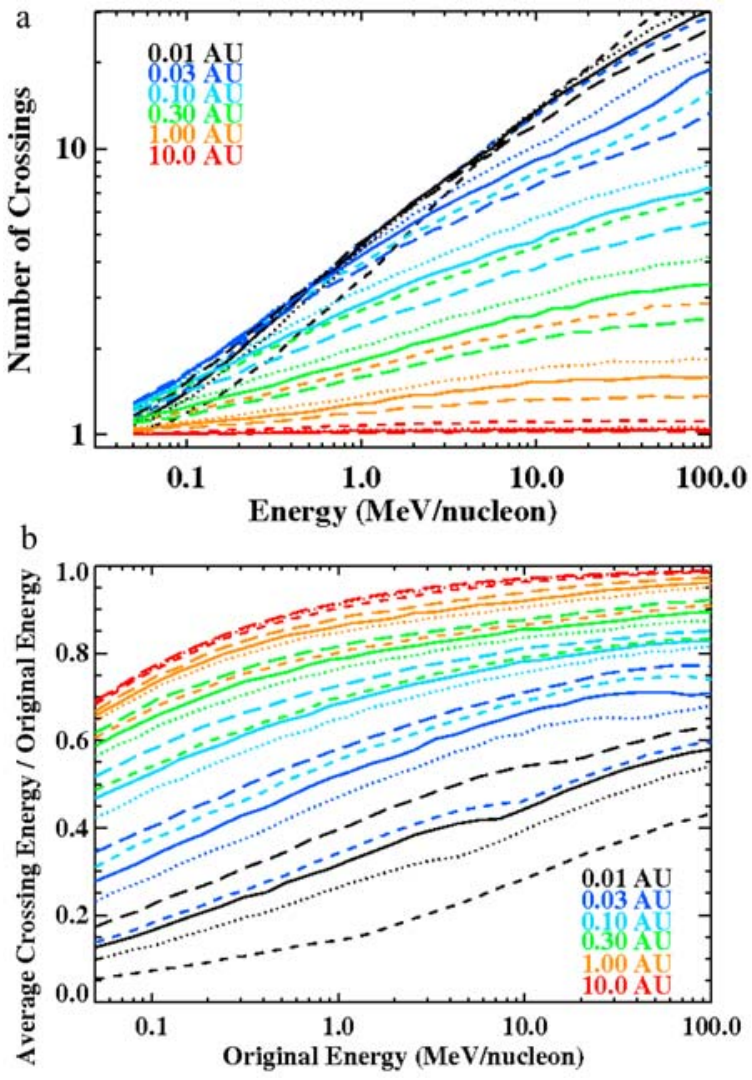

Figure 2. (a) Mean number of $1 \mathrm{AU}$ crossings and (b) the ratio of the average crossing energy to the original energy for the constant mean-free path scheme. The long dashed lines are for iron $(M / Q=4)$, the solid lines are for oxygen $(M / Q=8 / 3)$, the dotted lines are for helium and carbon $(M / Q=2)$, and the short dashed lines are for protons $(M / Q=1)$. The different colors represent the different mean-free paths. 


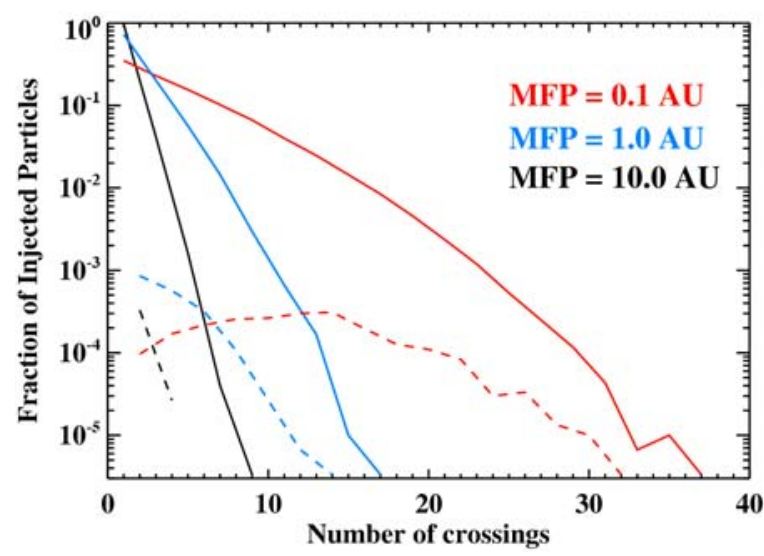

Figure 3. Fraction of the originally injected particles that cross a given number of times. This plot is solely for protons with an original energy of $1.52 \mathrm{MeV}$. The solid lines are for odd numbers of crossings, and the dashed lines are for even numbers of crossings. The different colors represent the different mean-free paths.

have been convected outward with the solar wind past $1 \mathrm{AU}$ after the full 10 days. This is reflected in the separation between the odd (solid lines) and even (dashed lines) number of crossings in this plot, though we did not include loss of particles impacting the solar surface.

[18] Figure 4 is similar to Figure 2, except using the schemes in equations (2) and (3) with $\lambda_{i}=0.1 \mathrm{AU}$. The constant and quasilinear schemes give similar results and dependencies in the number of crossings. However, the quasilinear mean-free path decreases with mass-to-charge ratio, the opposite dependence of the other two schemes. Since mean-free path is observed to increase with $M / Q$ for SEPs, this is probably unphysical, but the dependence on mass-to-charge ratio in all three schemes is weak. The equation (2) scheme $\left(\lambda_{\|} \sim \mathrm{r}^{2 / 3}\right)$ differs significantly from the other two, since the number of crossings begins to decrease for particles with original energies greater than about $1 \mathrm{MeV}$ nucleon $^{-1}$. Since a mean-free path that goes like $\mathrm{r}^{2 / 3}$ would physically represent a purely radial field, it is probably unrealistic, and the actual number of crossings the particles will see is probably closer to the constant or quasilinear values. With respect to energy loss, the constant scheme differs somewhat from the other two for particles with low original energies, but all three schemes have a similar qualitative dependence on original energy and the values become very similar for particles with high original energies. No simple summation of these schemes can be made, so it is important to examine the simulation results directly when making inferences about the source region.

\section{Application to a Large SEP Event}

[19] We applied these results to an impulsive flare-related SEP event beginning on 20 February 2002. Around 0612 UT on 20 February 2002, NOAA's GOES satellite observed an $\mathrm{X}$-ray class M5 flare at N12W72. The resulting solar energetic particle event was particularly large for a ${ }^{3} \mathrm{He}$-rich event, with a peak $5-10 \mathrm{MeV}$ nucleon ${ }^{-1}$ intensity of $0.5 \mathrm{He}$ $\left(\mathrm{cm}^{2} \mathrm{~s} \mathrm{sr} \mathrm{MeV}^{-1}\right.$ nucleon $^{-1}$. The particle event had an $\mathrm{Fe} / \mathrm{O}$ ratio at $>10 \mathrm{MeV}$ nucleon ${ }^{-1}$ of 0.63 , about 3.5 times the coronal value [Feldman and Widing, 2003], and the ${ }^{3} \mathrm{He} /{ }^{4} \mathrm{He}$ ratio at $\sim 7 \mathrm{MeV}$ nucleon ${ }^{-1}$ was $0.0415 \pm 0.0061$, suggesting the event was flare associated rather than $\mathrm{CME}$ associated. Furthermore, the relative timing of the energetic particle event and the flare suggest a release height of $<1.5$ solar radii [Mewaldt et al., 2003], unusually low for a CMEassociated event. Though the flare was accompanied by a CME with a speed of roughly $950 \mathrm{~km} \mathrm{~s}^{-1}$, the ion composition and timing analysis suggest this was a flare-associated event. Both the ion event and the accompanying electron event were initially highly "beamed" [e.g., Haggerty and Roelof, 2002] with a very anisotropic pitch angle distribution.

[20] Figure 5a presents the measured ion intensity for this event from EPAM, with the particles from the front lowenergy magnetic spectrometer (LEMS) 30 telescope (pointed generally sunward) and back LEMS 120 telescope (pointed generally antisunward) directions plotted separately. The event shows the earliest arriving particles arriving at the front, with the back particles arriving a few hours later, and the distribution becoming isotropic about 1 day after the event begins. A plot of the sunward and antisunward crossings from the simulation (Figure $5 \mathrm{~b}$ ) using the quasilinear mean-free path with $\lambda_{i}=0.1$ shows a qualitatively similar temporal
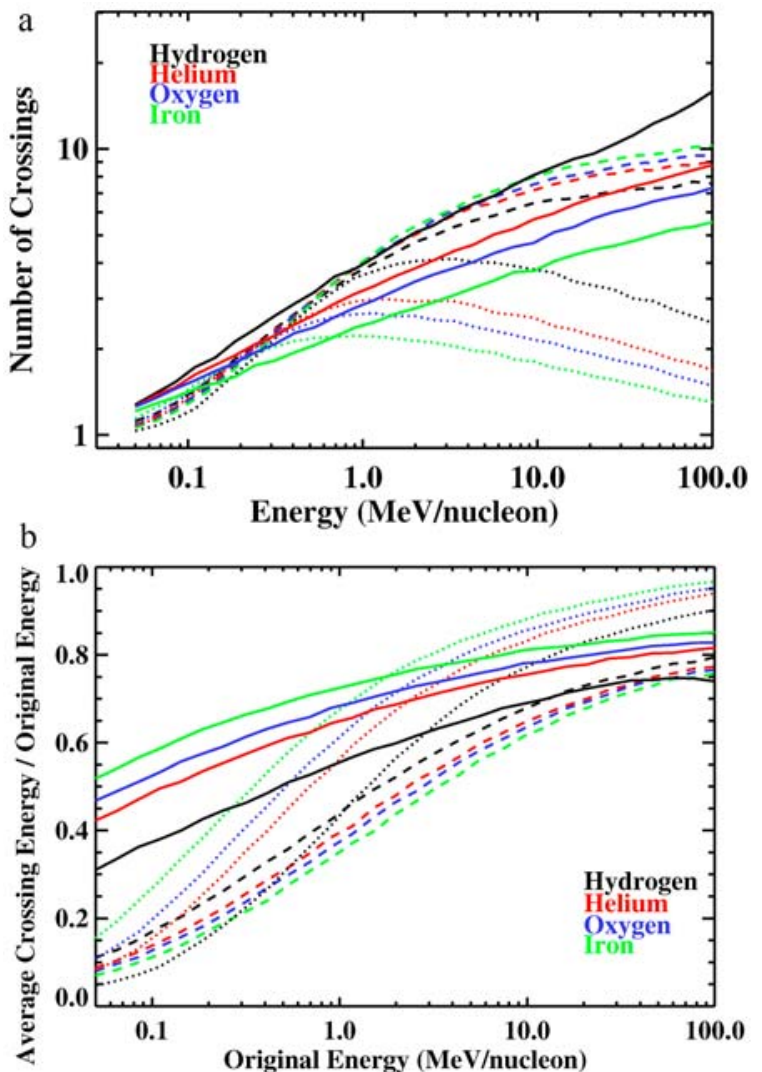

Figure 4. (a) Mean number of $1 \mathrm{AU}$ crossings and (b) the ratio of the average crossing energy to the original energy. The solid lines are for the constant mean-free path, the dotted lines are for the scheme in equation (2), and the dashed lines are for the quasilinear scheme. For each scheme, the base mean-free path $\left(\lambda_{i}\right.$ from equations (1), (2) and (3)) is $0.1 \mathrm{AU}$. 

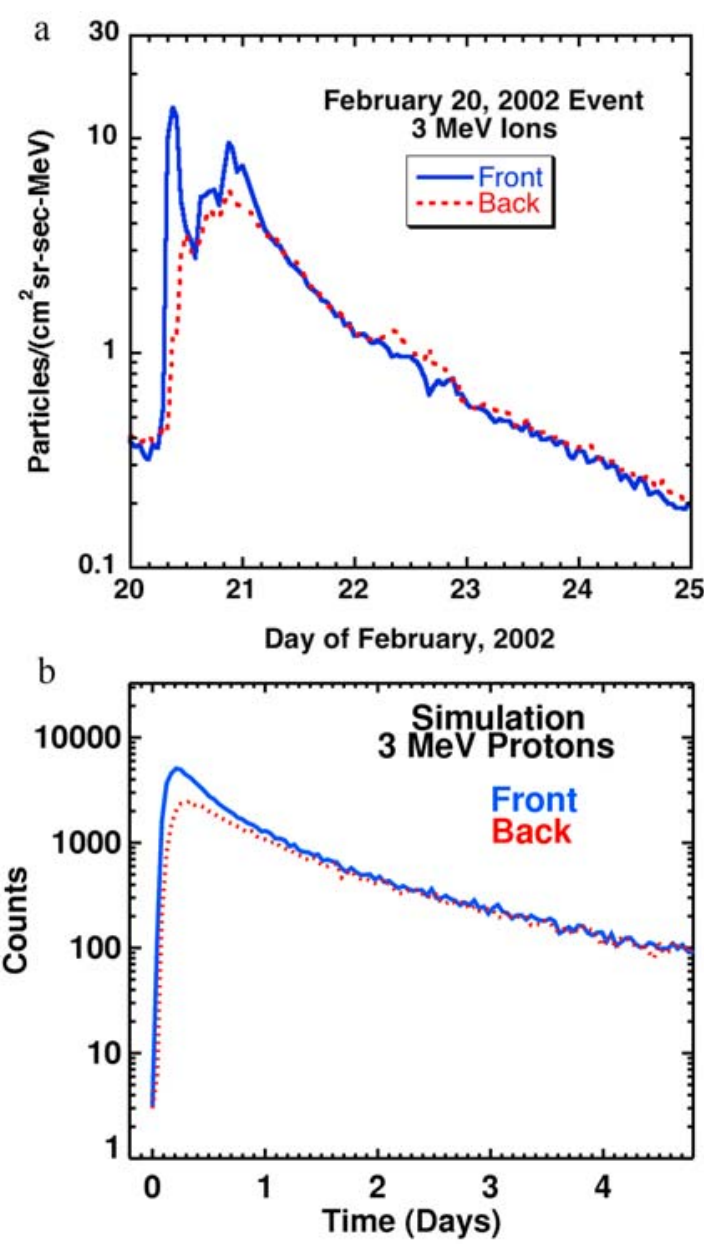

Figure 5. Intensity of the front (antisunward streaming) and back (sunward streaming) particles for the (a) February 2002 event and from (b) the simulation. The event data comes from LEMS 30 and LEMS 120 telescopes in EPAM. The simulation and observations are qualitatively similar, excluding the dropout in the event profile caused by a crossing of the heliospheric current sheet.

profile. The decrease in intensity starting at approximately 1000 UT on February 20 is associated with increased scattering from a heliospheric current sheet crossing, which our simulation does not include. From the simulation results presented in Figures 2 and 4, we expect the average number of crossings of $1.5 \mathrm{MeV}$ protons to be on the order of 5, so the back fluence and front fluence should be roughly comparable, and they are in both the simulated and observed fluences.

[21] Figure 6 presents the measured fluence (time-integrated differential intensity) spectra for $\mathrm{H}, \mathrm{He}, \mathrm{O}$, and $\mathrm{Fe}$ from three ACE instruments (ULEIS, EPAM, and SIS), with additional $\mathrm{H}$ and $\mathrm{He}$ measurements taken from GOES 8. These spectra were fit with an Ellison-Ramaty function [Ellison and Ramaty, 1985] that was modified to fit the low-energy rollover. The power law portions of the spectra are very similar in shape between species, though (as typically observed, e.g., by Mewaldt et al. [2005a]) the protons roll over at a higher energy than the heavier species.
[22] Using the results of the simulation, the measured fluence spectra were then used to find the energy required to accelerate the particles back at the Sun. The measured energy per square centimeter derived from the fluence spectrum at $1 \mathrm{AU}$ is divided by the average number of crossings as a function of the original energy and by the energy-loss fraction of original energy, assuming all particles of a given original energy have experienced the average energy loss. The result is multiplied by an assumed area that the event covers (a cone with a half angle of $20^{\circ}$ and uniform intensity). The resulting source strengths in ergs as a function of mean-free path are presented in Figure 7. The dependence on mean-free path is relatively weak because of competing effects in the simulation. As the amount of scattering increases, a larger proportion of the energy is lost and the number of crossings increases, so these corrections tend to cancel each other [Mewaldt et al., 2008].

[23] Figure 7 also shows the effect of using radially dependent mean-free paths on the derived source strengths. With the same base mean-free path $\left(\lambda_{i}\right)$, radially dependent schemes give slightly higher source strengths for all species. Because the measurement uncertainties on the fluence spectrum are relatively small, the uncertainty on the total energy per area near Earth can be reasonably estimated as $15 \%$ or less. However, changing the assumed area will have a large effect on the inferred source strength: with a $25^{\circ}$ cone instead of $20^{\circ}$, the estimated energy content increases by $55 \%$. Though the uncertainties of the values in Figures 2 and 4 cannot be calculated directly, the source strength results in Figure 7 are all within 30\% of the mean derived from all scattering schemes and assumed mean-free paths.

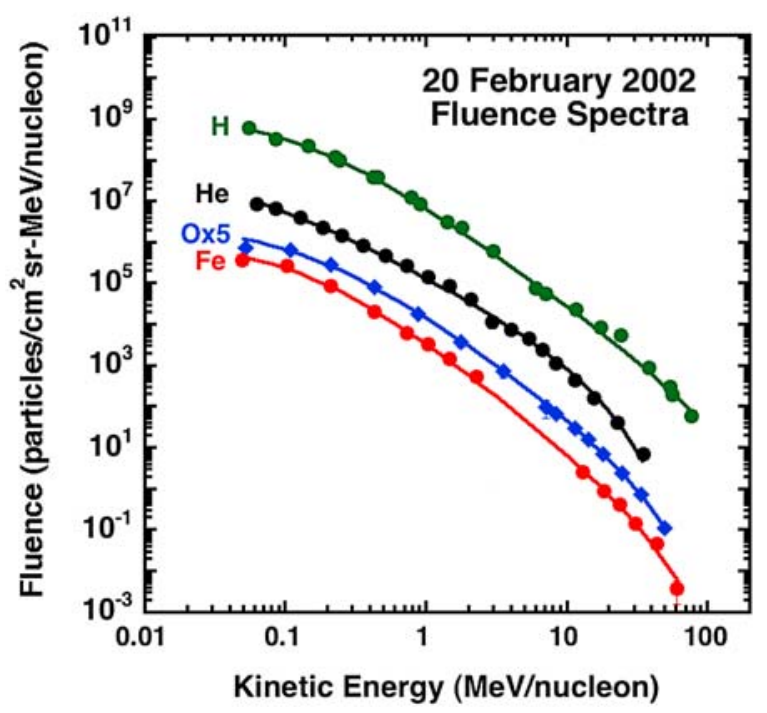

Figure 6. Fluence spectra for $\mathrm{H}, \mathrm{He}, \mathrm{O}$ (multiplied by 5), and $\mathrm{Fe}$, integrated over the 2 February 2002 solar energetic particle event. The proton spectrum is based on ACE EPAM, ACE ULEIS, and GOES 8 data. The helium spectrum is based on ULEIS, ACE SIS, and GOES 8 data, and the $\mathrm{O}$ and $\mathrm{Fe}$ spectra are based on ULEIS and SIS data. The spectra were fit with a spectral shape of the form $d J / d E=F\left(E+E_{i}\right)^{\gamma} \exp \left(-E / E_{o}\right)$, where the constants $F, E_{i}, \gamma$, and $E_{o}$ were allowed to vary from species to species. 


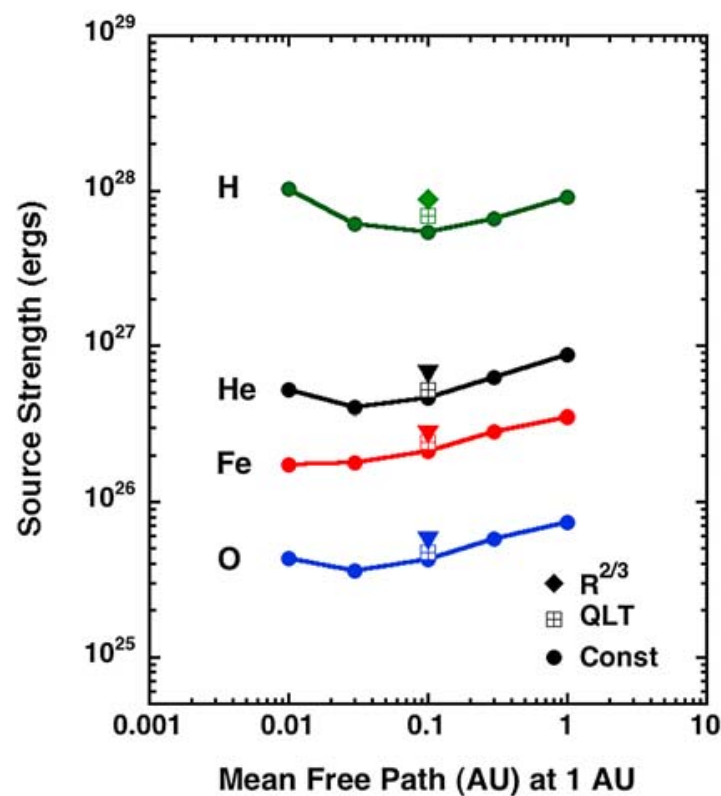

Figure 7. Derived particle source strengths as a function of mean-free path. The different species are indicated by color. The constant scheme was run for all mean-free paths, while the quasilinear and $\mathrm{r}^{2 / 3}$ are only presented for $\lambda_{i}$ of $0.1 \mathrm{AU}$, a typical mean-free path.

[24] Overall, these results suggest that past results derived from simple assumptions about the scattering mean-free path probably underestimated the source strength if they did not take into account the effects of adiabatic energy loss [e.g., Mewaldt et al., 2005a, 2005b], but when this correction is included (as in the work by Mewaldt et al. [2008] and this work) the exact form of the scattering scheme or magnitude of the mean-free path is ultimately less important than the assumed longitude and latitude distribution of the energetic particles. Multispacecraft studies by STEREO and nearEarth spacecraft such as ACE to determine these distributions more precisely would be extremely valuable for determining more precise estimates of the energetic particle source strengths.

\section{Summary and Conclusions}

[25] We have studied the transport of energetic particles in the inner heliosphere by simulating the trajectories of individual particles with a wide range of energies and a variety of species. We used three different schemes for the scattering mean-free path, one in which $\lambda_{\|}$is constant, one in which $\lambda_{\|} \sim \mathrm{r}^{2 / 3}$ (where $\mathrm{r}$ is heliocentric distance), and one in which $\lambda_{\|}$is based on quasilinear theory. By integrating the trajectories of a large number of individual particles, we have determined the average number of times particles at various energies cross $1 \mathrm{AU}$ and the average energy loss they experience during transport, which can be used to relate measured and actual properties of the particle population.
We find that mean-free path, particle species, and original energy of the particle all play significant roles in determining the number of crossings and energy loss in ways that cannot be easily summarized. Using scattering schemes with different dependencies in the mean-free path can also significantly affect the average number of crossings and energy loss. However, when applying these results to determining the energy content of an event, we find that an increased number of crossings is largely compensated for by the increased energy loss due to more scattering. By correcting for the number of times particles cross $1 \mathrm{AU}$ and for adiabatic energy loss in the manner described above, the derived source energy for the 20 February 2002 event is the same within less than a factor of 2 regardless of the mean-free path or radial dependence of the scattering scheme.

[26] Acknowledgments. We have greatly benefited from useful discussions of this topic with Randy Jokipii. We appreciate the availability of EPAM, SIS, and ULEIS data from the ACE Science Center and the availability of GOES data from NOAA. The work at University of Arizona was supported in part by NSF under grant ATM0447354. The work at Caltech was supported by NASA under grants NNX8AI11G and NNX06AC21G

[27] Amitava Bhattacharjee thanks Dennis Haggerty and A. Gordon Emslie for their assistance in evaluating this paper.

\section{References}

Bieber, J. W., W. H. Matthaeus, W. Smith, W. Wanner, M.-B. Kallenrode, and G. Wibberenz (1994), Proton and electron mean free paths: The Palmer consensus revisited, Astrophys. J., 420, 294-306.

Ellison, D. C., and R. Ramaty (1985), Shock acceleration of electrons and ions in solar flares, Astrophys. J., 298, 400-408.

Emslie, A. G., et al. (2004), Energy partition in two solar flare/CME events J. Geophys. Res., 109, A10104, doi:10.1029/2004JA010571.

Feldman, U., and K. G. Widing (2003), Elemental abundances in the solar upper atmosphere derived by spectroscopic means, Space Sci. Rev., 107, $665-720$.

Haggerty, D. K., and E. C. Roelof (2002), Impulsive near-relativistic solar electron events: Delayed injection with respect to solar electromagnetic emission, Astrophys. J., 579, 841-853.

Jokipii, J. R. (1966), Cosmic-ray propagation. I. Charged particles in a random magnetic field, Astrophys. J., 146, 480-487.

Li, G., G. P. Zank, and W. K. Rice (2003), Energetic particle acceleration and transport at coronal mass ejection-driven shocks, J. Geophys. Res., 108(A2), 1082, doi:10.1029/2002JA009666.

Mewaldt, R. A., et al. (2003), Heavy ion and electron release times in solar particle events, Proc. Int. Conf. Cosmic Rays 28th, 6, 3313-3316.

Mewaldt, R. A., et al. (2005a), Proton, helium, and electron spectra during the large solar particle events of October-November 2003, J. Geophys. Res., 110, A09S18, doi:10.1029/2005JA011038.

Mewaldt, R. A., et al. (2005b), What fraction of the kinetic energy of coronal mass ejections goes into accelerating solar energetic particles? Proc. Int. Conf. Cosmic Rays 29th, 1, 129-132.

Mewaldt, R. A., et al. (2008), How efficient are coronal mass ejections at accelerating solar energetic particles?, in Particle Acceleration and Transport in the Heliosphere and Beyond: 7th Annual Astrophysics Conference, AIP Conf. Proc., 1039, 111-117.

Parker, E. N. (1965), The passage of energetic charged particles through interplanetary space, Planet. Space Sci., 13, 9-49.

E. E. Chollet and R. A. Mewaldt, Space Radiation Laboratory, California Institute of Technology, 1200 E. California Blvd., Pasadena, CA 91125, USA. (echollet@srl.caltech.edu)

J. Giacalone, Lunar and Planetary Laboratory, University of Arizona, 1629 E. University Blvd., Tucson, AZ 85721, USA. 